\title{
Toward a multifaceted understanding of plants' adaptation mechanisms
}

\author{
Michitaro Shibata ${ }^{1} \cdot$ Junro Mogami $^{2}$ \\ Published online: 27 March 2019 \\ (c) The Botanical Society of Japan and Springer Japan KK, part of Springer Nature 2019
}

As sessile organisms, plants have developed adaptation mechanisms for changing environments. To deal with environmental problems facing the world today such as global warming and desertification, understanding plant adaptation mechanisms to environmental stresses is essential. Thus, researchers have been investigating the mechanisms of plant adaptation from various angles. This JPR Symposium entitled "Toward unveiling plant adaptation mechanisms to environmental stresses" reviews two different facets of plant adaptation mechanisms.

Shibata and Sugimoto (2019) review how environmental signals are transduced and altered root hair development. They point out that a gene regulatory network (GRN) consisting of transcription factors enables plasticity during root hair development. Especially, they claim that a GRN enables the plant to respond accordingly to multiple environmental signals. Shibata and Sugimoto (2019) also introduce the RSL4-GTL1 module as the core GRN for root hair development based on their recent findings (Shibata et al. 2018).

Scharwies and Dinneny (2019) broadly summarize current knowledge regarding water transport, perception and response in plants. First, they explain from a biophysical perspective how water moves from the soil through the plant and eventually evapotranspirates into the atmosphere. Secondly, they describe systemic and local responses to water deficit gleaned from a broad array of studies ranging from classical observations by Charles Darwin to the latest publications. Finally, Scharwies and Dinneny (2019) discuss putative water sensing mechanisms, particularly how wateravailability changes are transduced as chemical signals.

For future research, we emphasize the importance of further understanding systemic responses as well as local responses, both of which underlie harmonized and flexible plant response toward continuously changing environmental conditions. In addition to focusing on an individual study, it will be necessary to integrate pieces of findings to reveal the whole picture of plant adaptation mechanisms, which will accelerate providing solutions for coping with global-scale environmental problems.

\section{References}

Scharwies J, Dinneny JR (2019) Water transport, perception, and response in plants. J Plant Res. https://doi.org/10.1007/s1026 5-019-01089-8

Shibata M, Sugimoto K (2019) A gene regulatory network for root hair development. J Plant Res. https://doi.org/10.1007/s1026 5-019-01100-2

Shibata M, Breuer C, Kawamura A et al (2018) GTL1 and DF1 regulate root hair growth through transcriptional repression of ROOT HAIR DEFECTIVE 6-LIKE 4 in Arabidopsis. Development 145:dev159707. https://doi.org/10.1242/dev.159707

Publisher's Note Springer Nature remains neutral with regard to jurisdictional claims in published maps and institutional affiliations.

Michitaro Shibata

michitaro.shibata@ riken.jp

1 RIKEN Center for Sustainable Resource Science, Yokohama 230-0045, Japan

2 Graduate School of Agricultural and Life Sciences, The University of Tokyo, Bunkyo-ku, Tokyo 113-8657, Japan 